\title{
On missing meningiomas
}

\author{
AHMED EL-BANHAWY, P. W. E. SHELDON, AND \\ JOE PENNYBACKER
}

From the Department of Neurological Surgery, Radcliffe Infirmary, Oxford

It is well known that not all meningiomas can be removed completely, that if they are not removed completely they will cause further trouble, and that even some thought to have been removed completely will recur (Simpson, 1957). And yet the fact remains that they have about the most favourable prognosis of all intracranial tumours, and their differentiation from other kinds of neoplasm is therefore of great practical importance. A surgeon will usually approach a tumour known to be a meningioma with the intention of removing it as completely as can be done with safety. If, on the other hand, he knows the tumour to be an infiltrating glioma, he may feel that biopsy, or partial removal, with a decompression and radiation treatment subsequently, has as much to offer.

The clinical features are rarely adequate for this differentiation, and in the majority of cases help must be sought in special investigations, of which radiography is the most important. There is no doubt that most supratentorial meningiomas can be identified on a combination of plain radiographs, angiograms, and air encephalograms, but sometimes even with all three of these techniques a precise diagnosis cannot be made (Wickbom and Stattin, 1958). This report is concerned with 11 patients who had meningiomas which for a time eluded identification and were regarded as being gliomas. In nine cases the correct diagnosis was established at a second operation or in the late stages of the first one; one patient died without any attempt at veri-

TABLE I

THE CASES OF MENINGIOMA STUDIED

\begin{tabular}{lcc} 
Site & Total No. & 'Missed' Cases \\
\hline Posterior fossa & 31 & - \\
Subtemporal (middle fossa) & 13 & 1 \\
$\begin{array}{l}\text { Sphenoidal ridge } \\
\quad \text { Global }\end{array}$ & 50 & 5 \\
$\quad$ En plaque & 11 & - \\
Suprasellar & 17 & 2 \\
Olfactory groove and subfrontal & 19 & 1 \\
Convexity & 66 & 1 \\
Parasagittal and falx & 101 & 1 \\
Intraventricular & 6 & -11 \\
Miscellaneous & 8 & 11
\end{tabular}

fication; and one died from an unrelated cause 17 years after an operation at which an error in diagnosis was made. These cases occurred in a series of 322 meningiomas (Table I) and among some 10,000 patients with brain tumour syndromes investigated in the Department of Neurological Surgery in Oxford over a period of 22 years. The incidence of this problem is thus not high, but it will be apparent from what follows that there may be other cases in our material which have not yet come to light.

\section{THE CLINICAL PROBLEM}

In general terms the problem is this. A patient known to have an intracranial tumour, but there nothing in the clinical state, plain radiograph examination of the cerebrospinal fluid, electro encephalogram, etc., to tell with certainty what kind of tumour it is. An angiogram is done, and this shows the displacement of blood vessels associated with a tumour in a certain site, but there is no pathological circulation in the tumour area to point to its nature; all that can be said is that there is an avascular space-occupying lesion in the appropriate site. If the clinical features suggest a rapidly growing tumour, the surgeon may content himself with a biopsy through a burr hole, and this may establish the diagnosis; but in some cases the pathologist can only say that there are abnormal cells which may represent a glioma or may be a glial reaction around a tumour. The surgeon may then do a craniotomy and find no evidence of a tumour on the surface of the brain but an appearance of local swelling (broadening and pallor of convolutions) which he interprets as the surface manifestation of an intrinsic tumour such as a glioma. He then incises this pathological-looking area and finds oedematous tissue which looks gliomatous but on histological examination is not definitely so. The wound is then closed with a decompression, and the patient may be given radiation treatment subsequently on the assumption that the lesion is a glioma. Some months, or years, later various circumstances may 
lead to a review of the evidence, the investigations are repeated and a meningioma is discovered.

The following cases illustrate these points.

CASE 1 A woman, aged 44 (R.I. 261238), for one year before admission had suffered from headache, occasional generalized epileptic attacks, and progressive aphasia. On examination, she was well preserved both physically and mentally, and the positive findings amounted to bilateral papilloedema, an incomplete right homonymous hemianopia, expressive aphasia, and slight weakness of the right side of the face and the right upper limb. Radiographs of the skull showed rarefaction of the dorsum sellae and a left carotid angiogram indicated a mass in the fronto-temporal region without pathological circulation but thought from the displacement of vessels to point to a pterion meningioma. A left fronto-temporal osteoplastic flap was reflected and there was no meningioma at the pterion. The brain was tense, but it was possible to inspect the anterior part of the middle fossa and the lateral half of the sphenoidal wing and no tumour was seen. The postero-inferior part of the frontal lobe was pathological: the convolutions were specifically broad and pale and felt soft to the touch. An incision was made and the subcortical white matter was very soft and oedematous, but deeper in it felt abnormally firm. A portion was taken for histological examination and this was reported as being either gliotic brain or a low-grade astrocytoma. On this evidence the patient was given radiation treatment after operation, and for about 18 months she did very well: headache was relieved, her speech improved, and the epilepsy was less insistent. Indeed, for this time she lived a normal life. She then began to deteriorate rapidly with progressive aphasia, right hemiplegia, and sensory loss down the right side of the body. In this state she was readmitted two years after the first operation, the carotid angiogram was repeated and now showed unequivocally a tumour at the medial end of the sphenoidal wing, and this was removed: it proved to be a small meningioma, $2.5 \mathrm{~cm}$. in diameter, attached to the medial end of the sphenoidal wing just lateral to the optic nerve. Intravenous urea was given during this operation, and the brain was so slack that it was possible to remove the tumour without excising or bruising the frontal or temporal lobes. Even so, there was very little improvement: the aphasia persisted at a disabling level, and although she became ambulant, the right upper limb was useless, and she died from pneumonia eight months after operation.

There was a happier issue in the second case.

CASE 2 K.P. (R.I. 208026), a nursing sister, aged 42, was admitted on 14 May 1955, complaining of severe headache. For two years she had had headache on waking in the morning, and this was associated with a swishing noise in her head synchronous with the pulse. The headache would ease as the day went on, and did not seriously interfere with her duties, until eight days before admission, when she had a sudden violent pain over the right eye, and felt faint. She was admitted to another hospital where her account of an intracranial bruit and sudden exacerbation of headache led to the suspicion of an intracranial haemorrhage. There were no neurological abnormalities, however, and the spinal fluid was clear and colourless, although at a pressure of over $300 \mathrm{~mm}$. of water. The fluid contained $65 \mathrm{mg}$. of protein per $100 \mathrm{ml}$. Because of the persistent headache she was transferred to the Radcliffe Infirmary for further investigation.

On examination, she looked well but seemed to have severe headache and was naturally somewhat worried. There was no aphasia or any mental impairment. There was early papilloedema but no other neurological abnormalities, in particular no anosmia. Radiographs of the skull showed some rarefaction of the dorsum sellae. As the acute pain had been predominantly right-sided a right carotid angiogram was done, and this showed displacement of the middle-line vessels towards the right side. All of the evidence was interpreted as favouring an intrinsic tumour of the left frontal lobe. A left frontal osteoplastic flap was reflected. The convolutions in the anterior part of the frontal lobe were broad and pale and felt very soft as though there was an underlying cyst. Indeed, two or three small subcortical cysts, $0.5 \mathrm{~cm}$. in diameter, were seen on the surface; when pricked these contained clear colourless fluid. Aspiration of the soft area produced no cystic fluid, so an incision was made and carried to a depth of $5 \mathrm{~cm}$. The white matter was very soft and diffluent, indeed almost liquefied. We were uncertain whether this was simply oedema or a glioma, and the pathologist reported on a smear preparation that it was possibly gliosis or diffuse astrocytoma. While awaiting this report, the outer end of the sphenoidal wing and the anterior part of the anterior fossa were explored, and no meningeal tumour was seen, although the tension of the brain was such that a full exploration would not have been possible without excising some of the frontal lobe. The wound was closed with the provision of a subtemporal decompression. As there was some uncertainty about the pathological diagnosis, radiation treatment was withheld.

In most respects this patient did well. The headache was relieved, the papilloedema subsided, there was no epilepsy, no aphasia, nor any palpable neurological deficit. But there was a gradual and progressive impairment of her intellect and temperament: she was able to carry on nursing for six years, but in posts of diminishing responsibility, and by the end of 1961 she no longer felt competent for nursing and took a job as inspector in a small factory. Here too she had difficulty because of being shorttempered and forgetful. And so, as it seemed that she would soon be unemployable, her case was reviewed in March 1962. As before, there were very few abnormalities. On a superficial level, her mentality seemed quite normal, and careful psychometric studies showed only some impairment of learning capacity. It was felt that some of her 'mental' difficulties were probably neurotic, determined by her knowledge of having had a cerebral illness and the fear of being unable to follow her profession. The physical examination now revealed complete anosmia on the left side, but the sense of smell was intact on the right side. There were no other neurological abnormalities. Radiographs of the skull showed increased density in the region of the left cribriform plate. The lumbar spinal 
fluid pressure was normal, but the fluid contained $155 \mathrm{mg}$. of protein. A left carotid angiogram confirmed the impression which had gradually dawned on us, i.e., that this patient had all along had a meningioma of the left olfactory groove.

We were interested in the psychiatric aspects of the case, and as there was no pressing indication to remove the tumour, we sent her to work as a nurse in a psychiatric hospital for further observation and study. She did this adequately for three months, although certain minor shortcomings were apparent and the general standard of her work was not up to the standard of a ward sister, which she had been before her illness. She also complained of frequent headache, and although the decompression area was indrawn and there was no other evidence of increased pressure, it was felt that the tumour should be removed. This was done through a bifrontal bone flap on 2 August 1962, seven and a quarter years after the first operation. The left frontal pole was excised, and this exposed a spherical meningioma, $4.5 \mathrm{~cm}$. in diameter attached to the left olfactory groove but extending for $1 \mathrm{~cm}$. under the falx into the right side of the anterior fossa. When it was removed there was an endostosis at the posterior end of the olfactory groove, removal of which opened the ethmoid air cells, and this defect was repaired with fascia lata. Convalescence was uneventful: there were no new neurological developments, and the patient professed a considerable improvement in her mentality. As her troubles had been so largely subjective before, it was difficult for us to assess this improvement, but when seen three months after operation, she had so largely regained her confidence that she looked forward to resuming her profession.

It is worth recording that examination of the portion of frontal lobe which was excised to expose the tumour showed that part of the cortex (probably at the site of the biopsy seven years before) was replaced by tough, cystic tissue which was spongy and yellow. Histologically, the white matter was gliotic with numerous swollenbodied astrocytes and large and small cystic spaces. The pathologist commented that it would be difficult to distinguish this tissue from a diffuse astrocytoma.

In each of these cases a small, medially placed basal meningioma had evidently produced gross oedema of the frontal lobe. The swelling of the frontal lobe was such as to cause symptoms and signs of increased intracranial pressure, and to produce abnormalities in ventriculograms and angiograms which were indicative of an intrinsic tumour. In each case the appearance of the frontal lobe at operation was consistent with an intrinsic tumour, and the pathological examination of tissue removed was suggestive of glioma, although not definitely so. Indeed, in the second case with knowledge of the correct diagnosis, the pathologist recorded his difficulty in deciding whether the changes in the frontal lobe overlying the tumour were simply those of gliosis or a diffuse astrocytoma.

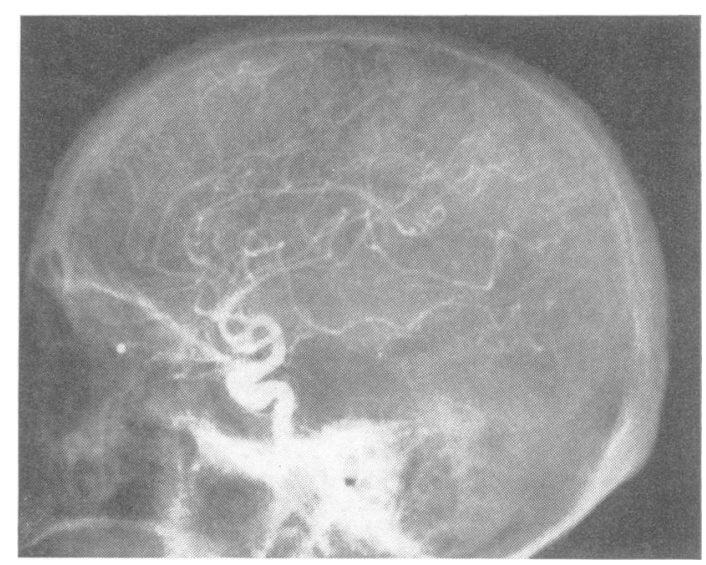

FIG. 1. Case 3. Left carotid arteriogram showing avascular mass in the left temporal region due to a small meningioma, $2 \mathrm{~cm}$. diameter, on the middle third of the sphenoidal wing.

The primary operation in both of these cases was done before the advent of intravenous urea. Older surgeons will be familiar with the technical problem which they presented, i.e., of a 'tight' frontal lobe in the dominant hemisphere, not greatly slackened? by tapping the opposite ventricle, and making exploration of the inner end of the sphenoidalo wing and the posterior part of the anterior fossa very difficult. The use of intravenous urea has largelyo done away with these difficulties, and it should at least be possible to make a thorough exploration in most cases. The third case demonstrates this point.

CASE 3 E.D., a woman aged 62 (R.I. 150484), had been in good health until 10 months before admission, when she began to be a little confused and to complain of noises in her ears. As time went on, her speech became slightly deranged, and she began to suffer from leftsided headache. On examination, there was early papilloedema, a slight expressive aphasia, and slight weakness of the right side of the face. Radiographs of the skull showed displacement of the pineal body to the right side and a left carotid angiogram showed pronounced upward displacement of the Sylvian group of vessels with no pathological circulation (Fig. 1). The evidence was taken as favouring a left temporal glioma, and at exploration, the convolutions in the anterior part of the temporal lobes were found to be broad, pale, and flattened. Intravenous urea had been given, however, and despite the local swelling of the temporal lobe, the brain generally was so slack that it was quite easy to elevate the temporal lobe and remove a small $(2 \mathrm{~cm}$. diameter) meningioma growing from the under surface of the middle third of the sphenoidal wing. This patient made a complete recovery.

In the cases described above it was focal oedema of the frontal or temporal lobe which led to mis- 
interpretation of the evidence. Another source of error is the presence of a large cyst in association with a meningioma. In one case, a cyst contained $85 \mathrm{ml}$. of clear yellow fluid and in another about $40 \mathrm{ml}$. Such large cysts are often taken as almost certainly indicating a glioma, but the knowledge that they can occur with meningiomas means that histological verification should be obtained. In one of these cases, an open exploration of the cyst in the temporal lobe disclosed a meningioma on the sphenoidal wing, and in the other the cannula used to evacuate a cyst in the frontal lobe met a firm resistance in its wall, and this led to a more thorough exploration and the removal of an olfactory groove meningioma.

Thus, both focal oedema and cysts in relation to a meningioma will produce the angiographic appearances of an avascular tumour. And it is hardly necessary to state that even when there is angiographic evidence of pathological circulation in a tumour, it may be misinterpreted: on many occasions, tumours with abnormal vascular patterns which we have regarded as glioblastomas or metastatic cancers have proved at operation to be meningiomas.

Yet another source of error is the occasional meningioma which looks and feels very much like a malignant glioma when exposed at operation. These are not common, but there was one in this series, which presented with the clinical features of a rapidly growing left frontal tumour, and the angiogram was interpreted as indicating a glioblastoma. At operation, the tumour presented on the surface of the frontal lobe, was poorly defined, and seemed to be invading rather than displacing the adjacent brain. A piece was taken for examination, and this was reported on as a glioblastoma, although when the tumour was removed, examination of the whole specimen left no doubt that it was a meningioma. Thus, the naked-eye identification of tumours can be very difficult, and we have on many occasions made the other mistake, i.e., of removing a circumscribed firm tumour, often attached to the dura, in the belief that it was a meningioma, only to learn from the pathologist that it was a glioblastoma.

The relevant findings in the cases not already mentioned, or to be described presently, can be summarized briefly. Two were characterized by epilepsy and increased intracranial pressure, with angiographic evidence of an avascular mass in the temporal lobe, and the presumptive diagnosis of glioma. Biopsy of the swollen temporal lobe in each case was reported as low-grade astrocytoma, but a further search revealed a sphenoidal wing meningioma in one case, and a small meningioma of the middle fossa in the other. In a third case, epilepsy and deterioration of memory led to an angiogram which was normal, but ventriculography indicated a mass in the left upper Rolandic region. This was explored and no meningioma was found, but the upper Rolandic region was swollen and was thought to harbour a glioma. Biopsy of this area was inconclusive. Over the next two years, a slowly progressive right hemiparesis led to re-exploration and the removal of a parasagittal meningioma.

It will be seen from Table I that with three exceptions, the 'missed' tumours were situated at the base of the brain, i.e., on the sphenoidal wing, in the olfactory groove or in the middle fossa. Of the exceptions, one was on the convexity of the frontal lobe, one was parasagittal, and one was situated in the right lateral ventricle. The clinical features and course of this patient were interesting and unusual enough to justify recording them.

CASE 4 A woman, aged 34 (R.I. 53704/46), was admitted on 23 February 1946 complaining of periodic throbbing headache for two years, and failure of vision in the right eye for 10 weeks. On examination, the abnormalities amounted to primary optic atrophy of the right eye, with vision reduced to finger-counting in virtue of a central scotoma. The left optic disc was swollen, the acuity was normal, and the field of vision full. There was no anosmia, nor indeed were there any other neurological abnormalities. The pressure of the spinal fluid was elevated and it contained $50 \mathrm{mg}$. of protein. Radiographs of the skull showed some thinning of the dorsum sellae and erosion of the right anterior clinoid process. These appearances, together with the Foster-Kennedy syndrome, made it likely that this patient had a meningioma at the medial end of the right sphenoidal wing. A right carotid arteriogram, however, showed an avascular right temporal lobe tumour. At a fronto-temporal exploration on 1 March 1946 the right sphenoid wing and middle fossa were carefully explored, and no extracerebral tumour was seen. The right temporal lobe was swollen, however, and an incision into it revealed soft, yellowish, honeycombed tissue, a portion of which was taken for examination and reported as being a glioma. The patient was left with a decompression, and had a course of deep $x$-ray therapy $(4,200 \mathrm{r})$ in the post-operative period.

She remained very well and led an active life for nearly 17 years until 29 December 1962, when she died suddenly from an apoplectic stroke involving the left cerebral hemisphere. At the necropsy, it was found that she had a congenital hydronephrosis of the left kidney, with hardly any cortex remaining. There was considerable hypertrophy of the left side of the heart. The left cerebral hemisphere was disrupted by a haemorrhage in its substance which had burst into the left lateral ventricle and the left subdural space. In the anterior part of the right lateral ventricle there was a firm ovoid tumour $(5 \mathrm{~cm} . \times$ $4 \mathrm{~cm}$.) which proved to be a fibroblastic meningioma. This was entirely intracerebral, and there was no growth in the skull base. The right temporal lobe showed the scar of the operation, but there was no evidence of neoplasm in it. 
There can be no doubt that it was the meningioma in the anterior part of the right lateral ventricle which caused this patient's symptoms 17 years before her death, although this is an unusual site for intraventricular meningiomas and the manifestations were also unusual. Presumably the local pressure effects of an intracerebral tumour caused the erosion of the right anterior clinoid process and the changes in the right optic nerve, but to explain the angiographic findings and the appearance of the temporal lobe at operation, we must suppose that the tumour in some way impaired the circulation in the temporal lobe. Nor can there be any doubt that her death was ultimately due to a cause unrelated to the tumour, i.e., an apoplectic stroke in the opposite hemisphere due to hypertensive vascular disease.

\section{DISCUSSION}

The extent and variability of the pathological changes in the brain around a meningioma are perhaps not fully realized although many authors have described them. McLean (1936) noticed that tumours of the brain sometimes show a rapid exacerbation of their effects because of oedema in the surrounding brain, and this he ascribed to venous stasis produced by local pressure of the growth on the draining veins. The temporary improvement following dehydrating measures or a decompression operation (as in cases 1 and 2 in the present series) he ascribed to decrease in the oedema. Scherer (1936) found that meningiomas frequently produced profound changes in the adjacent cortex and white matter, varying from classic oedema to coagulation necrosis. He attributed these changes to prolonged circulatory impairment, particularly impairment of venous drainage. Le Beau (1938) thought that the degree of oedema in relation to a tumour was a factor of its rate of growth, being most marked in glioblastomas and metastatic cancers, although at times quite profound in slowly growing tumours such as meningiomas. Surgeons have been aware of this for many years: there are occasional quite small meningiomas, e.g., of the convexity, which cause severe headache, gross papilloedema, and other manifestations of increased intracranial pressure out of all proportion to their size. At operation, the explanation is usually to be found in oedema of the brain around the tumour. Greenfield (1939) observed mild, diffuse oedema around meningiomas, and because it differed in degree in various areas in relation to the tumour, he thought it was not simply a local pressure effect, but was due to pressure on draining veins or to local effects of toxins and metabolites from damaged brain tissue or from the tumour itself. In a later study, he and his associates (Greenfield, Blackwood, McMenemy, $\underset{\mathbb{D}}{ }$ Meyer, and Norman, 1958) mention meningiomas as among the brain tumours commonly causing oedema, and they identified acute and chronic types, $\overrightarrow{0}$ which they thought were due to venous compression. T Noetzel (1951) drew a distinction between the meningiomas attached to the dura and those growing from the leptomeninx. The former derive their main blood supply from dural vessels, and only embarrass the adjacent brain by local pressure effects, $\overrightarrow{\bar{N}}$ i.e., by simple compression. The leptomeningeal $\frac{\pi}{0}$ growths, however, depend on pial vessels for their blood supply, and may to some extent deprive the adjacent cortex of blood leading to oedema and necrosis. It should be stated, however, that this explanation does not accord with the observed o findings in our cases, most of which were of small $\overrightarrow{0}$ basal tumours with a dense dural attachment, and $\vec{\overrightarrow{ }}$ were associated with profound oedema. These $\omega$ changes were seen in their entirety in a case which did not come to operation.

CASE 5 A man, aged 74 (R.I. 202389), had apparently o suffered from attacks of giddiness for a year beforg or admission to hospital, but there were no focal neurologica of abnormalities until four weeks before admission, whem $N$ he began to deteriorate rapidly with headache, weaknes 용 down the left side of the body, incontinence of urine, ang stupor. In this state he was admitted, when it was foure that there was also a left homonymous hemianopia The clinical evidence thus indicated a rapidly growing tumour in the right cerebral hemisphere. A right carotid angiogram showed a large avascular mass in the rig $\vec{\varphi}$ temporal lobe (Fig. 2), and because of the patient's age, $\stackrel{\omega}{\omega}$ poor general condition, and the probability that the tumour was a malignant one, no further investigation was done. He died two weeks after admission, and at necropsy there was a small meningioma of the right sphenoidal wing, $3 \mathrm{~cm}$. in its long axis (Fig. 3). The adjacent temporal lobe was soft and greatly expanded by $\mathbb{D}$ oedema: the right Sylvian fissure was elevated by nearly $2 \mathrm{~cm}$. When the brain was cut (Fig. 4) the oedema was obvious, and there were areas of necrosis with discoloration which could easily have misled a surgeon exploring the temporal lobe.

Some of the inferences to be drawn from a study of these cases are obvious, and are doubtless well known to many, but we think it worth enumerating the following points: Meningiomas may be associated with gross focal oedema of the brain in their neighbourhood. This is especially common in small tumours on the sphenoidal wing where obstruction of veins draining into the spheno-parietal and $D$ cavernous sinuses may cause focal oedema of the temporal and frontal lobes.

In the absence of the abnormal vascular pattern associated with meningiomas, angiograms may show only the displacements due to oedema, or in some 


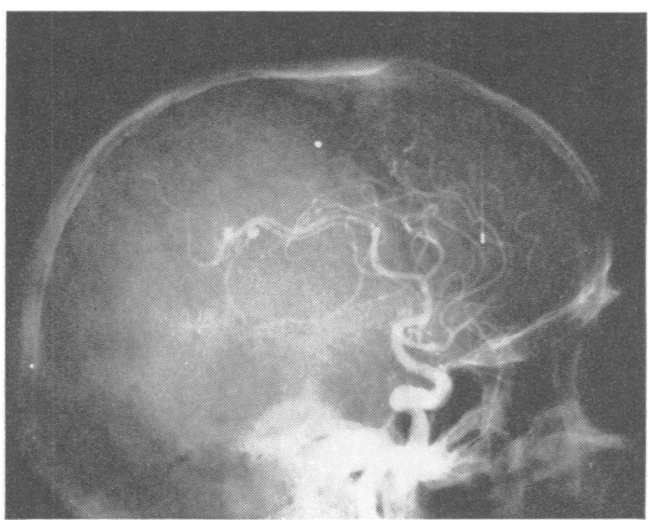

FIG. 2. Case 5. Right carotid arteriogram showing avascular swelling of the right temporal lobe due to the meningioma on the sphenoidal wing seen in Figure 3.

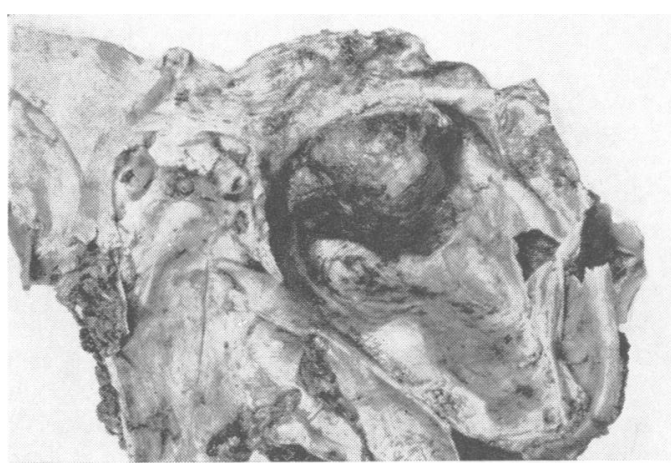

FIG. 3. Case 5. Meningioma of the medial end of the right sphenoidal wing.

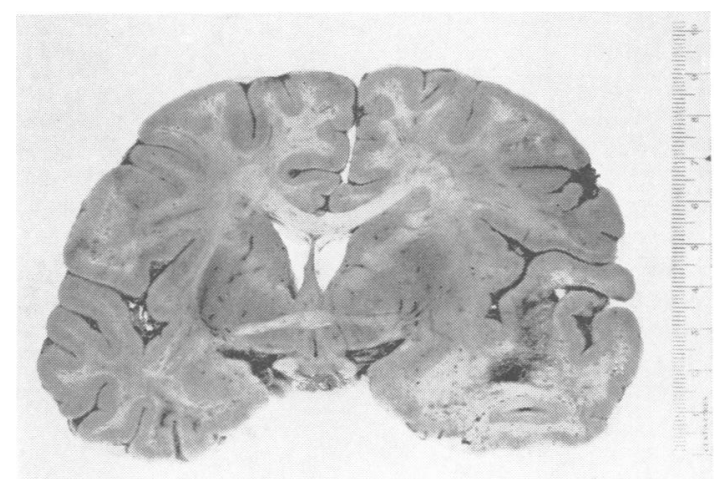

FIG. 4. Case 5. Oedema and necrosis of the right temporal lobe in association with the tumour seen in Figure 3. cases to a large cyst in relation to a meningioma. From a retrospective study of the angiograms in this series we found possible sources of error in the poor quality of the angiograms in two cases, both done over 10 years ago, and this risk has obviously been lessened with improvements in technique and contrast media. In two other cases, only internal carotid arteriograms were done. As meningiomas may derive a significant part of their blood supply from the external carotid circulation, an external or common carotid angiogram should be done in suspected cases, and especially where an internal carotid angiogram has shown an avascular temporal mass. Rapid serial angiography may also yield valuable information about later pathological circulation, such as obstruction of the deep middle cerebral vein, the basal vein, and spheno-parietal sinus, which might be missed in conventional exposures.

Focal oedema of the brain overlying a meningioma can simulate an intrinsic tumour in its external appearance, on incision, in rapid biopsy examination, and in formal histological studies.

Knowledge of the problems set out above should encourage the surgeon to carry out a very thorough search for the tumour, and there is no doubt that the use of intravenous urea has been of enormous help in this connexion.

The surgeon should be satisfied with nothing less than an absolute pathological diagnosis, and where this is lacking, the patient should be kept under observation, and the relevant investigations repeated as circumstances dictate.

\section{SUMMARY}

Eleven cases of meningioma mistaken for glioma are described. In the majority of cases, these were small basal tumours associated with gross focal oedema of the brain, giving rise to misleading angiograms.

The difficulty of distinguishing between focal oedema and intrinsic tumours is discussed.

The necessity of high-quality angiograms, a thorough search for the tumour, and absolute pathological verification is stressed.

\section{REFERENCES}

Greenfield, J. G. (1939). Brain, 62, 129.

- Blackwood, W., McMenemy, W. H., Meyer, A., and Norman, R. M. (1958). Neuropathology, pp. 100-103. Arnold, London. Le Beau, J. (1938). L'Oedème du cerveau: son rôle dans l'évolution des tumeurs et des abcès intra-crâniens (Thesis), p. 48. Créations Elbé, Paris.

McLean, A. J. (1936). In Handbuch der Neurologie, edited by 0 . Bumke and O. Foerster, vol. 14, pp. 131-285: Raumbeengende Prozesse. Springer, Berlin.

Noetzel, H. (1951). Beitr. path. Anat., 111, 391.

Scherer, H. J. (1936). Rev. neurol., 66, 307.

Simpson, D. (1957). J. Neurol. Neurosurg. Psychiat., 20, 22.

Wickbom, I., and Stattin, S. (1958). Acta radiol. (Stockh.), 50, 175. 